\title{
LUCIANO FLORIDI*
}

\section{Trois leçons philosophiques de Turing et la philosophie de l'information}

\section{Introduction}

Quand on se penche sur l'héritage philosophique de Turing, deux risques se posent. Le premier, c'est de le réduire à son test célèbre (Turing 1950). Ce qui a toutefois le mérite de la clarté. N'importe qui peut reconnaître la contribution en question et la situer dans le débat important sur la philosophie de l'intelligence artificielle. Le second risque est de le diluer dans un récit universel, faisant des idées de Turing les graines de tout ce que nous faisons et savons aujourd'hui. Ceci a l'avantage de reconnaître la grandeur de ce génie. Cependant, dans un cas comme dans l'autre, nous allons probablement moins identifier les contributions conceptuelles de Turing qui nous ont aidés à forger un discours philosophique contemporain et qui méritent d'être approfondies et développées. Pour éviter ces deux risques, je me concentrerai dans les pages suivantes sur trois leçons philosophiques spécifiques, qui me semblent particulièrement significatives pour l'émergence de la philosophie de l'information et ses développements. Je ne propose pas ici une analyse philologique ni savante, mais un exercice minimaliste, herméneutique. Façon de faire écho à la démarche de Turing et de rendre hommage à son génie extraordinaire : ses interprètes n'ont pas fini de tirer parti de son héritage intellectuel. Je souhaite qu'un jour Turing devienne aussi important que Frege dans notre canon philosophique.

Les trois leçons philosophiques sur lesquelles je voudrais attirer l'attention du lecteur se détaillent ainsi : 
a) comment le travail de Turing sur la méthode des niveaux d'abstraction (LoA) peut-il nous aider à poser correctement des questions philosophiques?

b) quelles questions philosophiques apparaissent les plus urgentes aujourd'hui, consécutivement au travail de Turing?

c) et enfin, quelle est l'influence de Turing dans la formation de notre nouvelle anthropologie philosophique - ce que j'appellerai la quatrième révolution ?

Je relierai ensuite ces leçons au développement de la philosophie de l'information, c'est-à-dire le domaine philosophique concerné par l'enquête critique sur la nature conceptuelle et les principes de base de l'information - y compris sa dynamique, son utilisation et ses avancées scientifiques et par l'application aux problèmes philosophiques des méthodes computationnelles (et théoriques) spécifiques de l'information. La philosophie de l'information s'appuie sur une interprétation explicite, claire et précise du classique « ti esti », à savoir la question : «Qu'est-ce que l'information ? », qui est la marque la plus claire d'un nouveau domaine de recherche. Comme souvent, une telle question fondatrice d'un domaine philosophique sert plus à délimiter une zone de recherche qu'à détailler ses problèmes spécifiques - que nous avons à peine commencé à dresser. Dans la conclusion, je soutiendrai que, même si Turing n’a jamais développé une philosophie de l'information, cette dernière serait inconcevable sans son héritage et sans les trois leçons décrites dans cet article.

\section{Leçon 1 : fixer le niveau d'abstraction ou comment poser les questions philosophiques?}

Imaginons le scénario suivant. Vous demandez le prix d'un objet à vendre, par exemple une voiture d'occasion et vous recevez la réponse suivante : 5.000. La question a concerné une variable, le prix $x$ de la voiture en question, vous avez reçu une valeur numérique exacte pour $x$, et pourtant quelque chose manque. Vous n'avez toujours aucune idée du prix, parce que vous ne connaissez pas le type de la variable : Renvoie-t-elle à des livres britanniques, des dollars américains, des euros ? Certes, habituellement, le contexte aide. Si vous êtes en Angleterre et si vous vous adressez à un concessionnaire d'automobiles, il supposera que votre question renvoie à un prix en livres britanniques et il en sera de même pour sa réponse. C'est un point de détail insignifiant, pouvez-vous penser. Dans un tel contexte, les maximes conversationnelles de Grice s'appliquent évidemment et la question de l'interprétation se résout d'elle-même. Pour autant, cette hypothèse implicite est cruciale, ce qu' on oublie trop souvent. En novembre 1999, la NASA 
a perdu la navette spatiale Climat de Mars (MCO), d'une valeur de 125 millions de dollars, tout simplement parce que les ingénieurs de Lockheed Martin ont utilisé les unités de mesure anglaises (aussi nommées « impériales »), tandis que l'équipe de l'agence a utilisé le système métrique pour une opération-clé de navigation spatiale. Du coup, le $M C O$ est entré en collision avec Mars ${ }^{1}$. Le fait de supposer que les contextes désambiguïseront toujours les types des variables induit le risque d'erreurs coûteuses. Cette digression a-t-elle un lien avec Turing, pouvez-vous demander ? Pour montrer que oui, je présenterai un modèle légèrement abstrait d'information factuelle ${ }^{2}$.

Nous pouvons traiter les informations factuelles précédentes (le prix de notre voiture d'occasion) comme un composé d'une question + sa réponse. Au prix d'une simplification théorique, notre question peut être réduite à un booléen, suivi par un oui ou par aucune réponse. Dans la version originale de notre exemple, le modèle appliqué devient alors : [Le prix de cette voiture est-il de 5,000 ? + oui]. Nous voyons immédiatement que le problème ne se cache pas dans la réponse, mais dans la question : elle ne contient aucune indication du type de la variable dont il s'agit. L'information correcte est bien sûr : [Le prix de cette voiture est-il de $£ 5,000$ ? + oui]. Nous venons de présenter le niveau correct d'abstraction ou LoA, représenté par le symbole pour des livres britanniques (et non, par exemple, par le symbole des dollars, \$, ni par celui des euros $€$ ). Or, Turing a été le premier à comprendre l'importance cruciale d'exprimer le LoA auquel on peut poser des questions raisonnables. Cela pourrait sembler étonnamment évident, mais le deuxième exemple apporté ci-dessus, concernant le $M C O$, montre à quel point il est facile et dangereux d'oublier quel est le LoA implicite. Le fait qu'il soit important de préciser son propre niveau d'abstraction a souvent paru aussi évident que le fait que la terre soit ronde. Pourtant, nous voyons là le génie de Turing. Bien sûr, sa contribution ne fut pas d'introduire le concept de variables typées, ni d'avoir établi le besoin de systèmes de référence. Ces idées étaient déjà communes en son temps. Sa leçon fut de faire comprendre, pour la première fois, comment on pouvait aussi répondre à des questions philosophiques et conceptuelles en fixant précisément le niveau d'abstraction qui garantisse un sens à une réponse. C'est ici l'une des contributions les plus grandes et durables de son test célèbre (Turing 1950), plus importante que les prédictions ou conclusions négatives à tirer à partir du moment où les machines passeraient ce test, ou bien des conséquences qu'il faudrait en tirer au moment où elles l'auront vraiment passé (Floridi, Taddeo et d'autres. 2009).

On oublie parfois que Turing a lui-même refusé de fournir une réponse à la question « Une machine peut-elle penser ?», parce qu'il l'a considérée comme «trop vide de sens pour mériter 
une discussion ». En utilisant encore une fois notre exemple automobile, cela reviendrait à demander que le prix d'une voiture soit exprimé avec des chiffres absolus et sans qu'aucune monnaie ne soit spécifiée. C'est évidemment absurde. Nous comprenons alors l'objection de Turing : la question qui lui était adressée impliquait des concepts vagues tels que «la machine » et « la pensée ». Autrement dit, elle manquait d'un niveau clair d'abstraction (LoA). Donc il a suggéré de la remplacer par le jeu de l'imitation (Imitation Game), qui est précisément plus raisonnable et moins exigeant parce qu'il fixe un scénario sur la base de règles facilement applicables et contrôlables (Moor 2003). Ce faisant, Turing a spécifié un LoA - la «monnaie d'échange» qu'il a choisie pour le jeu était l'intelligence humaine, mais il pouvait y avoir quelque chose d'autre à la place, de l'intelligence animale à la créativité humaine, comme tant d'autres versions du jeu de l'imitation de Turing l'ont montré - et elles ont posé une nouvelle question, qui peut être résumée ainsi : «Peut-on conclure qu'une machine pense, au niveau d'abstraction représentée par le jeu de l'imitation ?». Après un demi-siècle, la philosophie est toujours en train de tirer les leçons d'une précision si cruciale ${ }^{3}$. Nous pouvons désormais nous tourner vers la deuxième leçon, qui exigera une prémisse plus longue.

\section{Leçon deux : se concentrer sur les problèmes les plus importants, ou bien quelles questions philosophiques sont à poser ?}

Le 23 avril 2010, Bill Gates a donné une conférence au MIT (Massachusetts Institute of Technology) dans laquelle il a demandé: « Les esprits les plus brillants se penchent-ils sur les problèmes les plus importants ? » Par « les problèmes les plus importants » il voulait évoquer «l'amélioration des conditions de vie des plus pauvres ; le développement de l'éducation, de la santé, de la nutrition ». Cette liste devait probablement inclure l'extension et l'affermissement de la paix, le respect des droits de l'Homme, l'amélioration des conditions environnementales et du niveau de vie, etc. Si nous suivons cette proposition, les esprits philosophiques les plus brillants devraient eux aussi orienter leur attention vers tous ces défis urgents. Il faudrait alors arrêter de philosopher et, au lieu de cela, commencer à agir concrètement pour ce monde désordonné. Autrement dit, nous pourrions fermer nos départements de philosophie et arrêter définitivement de corrompre philosophiquement les plus brillants de nos jeunes esprits. Pourtant, une telle solution apparaît comme un suicide ou une désertion. Elle s'apparente à la décision de brûler le panier de la montgolfière dans laquelle nous voyageons au prétexte que celle-ci descend trop vite. La philosophie correspond à ce que l'on doit garder dans un monde bon, et non à ce dont on doit se 
débarrasser dans un mauvais. Athènes est un endroit meilleur avec Socrate que sans. Ainsi doit-il y avoir une démarche différente à suivre que celle de la focalisation sans recul sur les problèmes les plus manifestes.

Le fait est que cette philosophie peut être extrêmement utile. C'est en effet la philosophie, conçue comme construction de concepts, qui forge et affine les nouvelles idées, théories, perspectives et, plus généralement, le cadre intellectuel qui peut être utilisé pour comprendre et traiter les questions en surplomb qui nous défient si urgemment. Dans l'effort collectif produit par les esprits les plus brillants, les esprits philosophiques peuvent apporter leur contribution grâce à des mises en perspective et des visions, des analyses et des synthèses, des démarches heuristiques et des méthodes qui toutes peuvent nous aider à simplifier ou solutionner ces «problèmes les plus importants ». Dans la bataille contre la stupidité, l'obscurantisme, l'intolérance, les fanatismes et les fondamentalismes de toutes sortes, la bigoterie, le préjugé et la simple ignorance, chaque effort est précieux. Si ce constat donne l'impression d'être autoréférentiel, il rappelle aussi que plus grand est espéré le bond en avant, plus grand doit être l'élan qui le précède. On pourrait dire, en restant dans le registre de la métaphore, que la philosophie s'occupe des racines de la plante afin que sa partie aérienne puisse grandir plus sainement.

Faisons le choix de prendre le rappel précédent comme une hypothèse raisonnable. Quelles idées, théories, perspectives et, plus généralement, quel cadre intellectuel les philosophes devraient-ils concevoir aujourd'hui et dans un futur proche pour que leur contribution soit opportune et utile? Quelles questions philosophiques devraient-ils aborder ? La réponse serait inconcevable sans l'héritage de Turing, car il est à la base des lignes conceptuelles qui traversent la plupart de nos «problèmes les plus importants ». Dans une société globale de l'information, la plupart des défis cruciaux auxquels nous faisons face sont liés à des informations et à des technologies de communication, en termes de causes, d'effets, de solutions, d'enquêtes scientifiques, d'améliorations réelles, de ressources conceptuelles qui ont été nécessaires pour les comprendre, ou même justement de la richesse exigée pour les aborder, comme la figure de Bill Gates le montre clairement. Évidemment, les ressources de l'information, les technologies et les sciences associées ne sont pas une panacée, mais elles constituent un instrument crucial dans notre combat contre tant de maux. Or elles n'existeraient pas sans Turing : sans sa conceptualisation de l'ordinateur et de l'automate.

La deuxième leçon qu'il nous faut apprendre de Turing consiste donc à prendre en charge conceptuellement, pour le meilleur comme pour le pire, les objets, les théories, les manières de 
voir induites par ses découvertes ${ }^{4}$, à faire le tri des questions que les esprits philosophiques devraient aborder. Les technologies de la communication et de l'information ont profondément changé beaucoup d'aspects de notre vie, y compris la nature de la communication, de l'éducation, le travail, le loisir, la production industrielle et les affaires, les services médicaux, les relations sociales et elles ont également armé des conflits. Elles ont une influence radicale et répandue sur nos vies morales et sur les débats éthiques contemporains. Les exemples viennent aisément à l'esprit : de la confiance en ligne à l'espionnage téléphonique, du digital divide à une « société de surveillance » dystopique, de la privacy et la liberté d'expression à Wikileaks, des amants virtuels à la cyber-guerre. Nous vivons dans une infosphère dans laquelle, derrière les problèmes les plus importants, se trouve souvent une machine de Turing. C'est un nouveau monde dans lequel nous avons commencé à re-conceptualiser nous-mêmes.

Cette seconde leçon en induit une troisième, toujours induite par Turing, et que je vais détailler dans la section suivante: ce nouveau monde nous invite donc à renouveler nos façons de conceptualiser.

\section{Leçon trois : le développement d'une nouvelle anthropologie philosophique ou de quelle perspective approcher les questions philosophiques ?}

En simplifiant à l'extrême, la science a deux façons fondamentales de changer notre compréhension du monde. L'une peut être appelée extrovertie, ou ouverte au monde, et l'autre introvertie, ou se rapportant à nous. Trois révolutions scientifiques ont eu un grand impact, tant de façon extrovertie qu' introvertie. Dans le changement de notre compréhension du monde externe et de la manière dont nous pouvons interagir avec lui, elles ont aussi modifié notre conception de qui nous sommes et de ce que nous pouvons espérer devenir. Après Copernic, la cosmologie héliocentrique a déplacé la Terre et par conséquent l'humanité du centre de l'univers. Darwin a montré que toute espèce de vie s'est développée, au fil du temps, en partant d'ancêtres communs, par sélection naturelle, déplaçant ainsi l'humanité du centre du royaume biologique. Et après Freud, nous reconnaissons, de nos jours, que l'esprit est aussi inconscient et soumis aux mécanismes de défense de la répression, le déplaçant ainsi du centre de la rationalité pure, une position qui avait été assumée comme indiscutable, au moins depuis Descartes. Le lecteur qui, comme Popper ou moi-même, serait réticent à suivre Freud dans sa description de la psychanalyse comme une entreprise strictement scientifique, telle que l'astronomie ou la théorie 
évolutionniste, pourrait néanmoins accepter l'idée que la neuroscience contemporaine soit un candidat prometteur à un rôle aussi révolutionnaire. Dans tous les cas, le résultat est qu'aujourd'hui nous reconnaissons que nous ne sommes pas immobiles ni au centre de l'univers (la révolution copernicienne), que nous ne sommes pas séparés ni différents du reste du règne animal (la révolution darwinienne) et que nous ressemblons peu à de purs esprits entièrement transparents à eux-mêmes (la révolution freudienne ou neuroscientifique).

On peut éventuellement mettre en doute une telle représentation de la science. Après tout, Freud lui-même (Freud 1917) était le premier à interpréter ces trois révolutions dans le cadre d'un seul processus de réévaluation de la nature humaine (Weinert 2009). Sa manœuvre herméneutique était, certes, plutôt égoïste sinon intéressée. Mais sa part de rationalité est indéniable. Notre intuition, quand nous évoquons l'idée d'une révolution numérique ou informatique, avec son lot de transformations profondes et significatives de la vie humaine, me semble exprimer un sentiment analogue : elle exprime elle-aussi une part de vérité, qui renvoie au même processus de dislocation et de réévaluation de la nature fondamentale de l'humanité et de son rôle dans l'univers que j'évoquais précédemment. C'est pourquoi je fais référence à une quatrième révolution, associée à la personne de Turing et aux conséquences de sa pensée telles qu'elles ont été mises en pratique à partir des années 1950. L'informatique et les applications technologiques résultantes ont eu une influence tant extrovertie qu'introvertie. Elles ont d'abord fourni des capacités épistémiques et d'ingénierie sans précédent pour comprendre et analyser des faits naturels et artificiels ; ce faisant, elles ont ensuite jeté une nouvelle lumière sur qui nous sommes, sur les façons dont nous sommes reliés au monde et par conséquent sur les façons que nous avons de nous comprendre et par suite sur ce que nous pourrions devenir. Aujourd'hui nous acceptons peu à peu l'idée que nous ne sommes pas (que) des entités autonomes et uniques, mais aussi des organismes informationnellement incarnés (inforgs), mutuellement connectés et incorporés dans un environnement que nous partageons avec des agents semblables à bien des égards, tant naturels qu'artificiels : l'infosphère. Turing a changé notre anthropologie philosophique autant et même plus que Copernic, Darwin et Freud ont pu le faire. Ceci a un impact significatif sur ce que signifie faire de la philosophie après Turing : c'est le dernier point sur lequel je voudrais attirer l'attention des lecteurs.

\section{Leçons apprises : l'établissement d'une nouvelle philosophie de l'information, ou comment saisir la signification du monde aujourd'hui ?}

Qu' est-ce qui permettrait à l'humanité de saisir la signification de notre monde contemporain, de 
le respecter et de l'améliorer effectivement, et par conséquent d'aider à la résolution « des problèmes les plus importants » ? La réponse semble tout à fait simple : une nouvelle philosophie de l'information. Parmi nos concepts banals et techniques, celui d'information est actuellement l'un des plus importants, largement utilisé, mais assurément le moins compris. Les esprits philosophiques les plus brillants devraient tourner leur attention vers ce concept, pour concevoir une philosophie de notre temps qui soit correctement conceptualisée pour notre temps. Un tel conseil peut être considéré - pour proposer un écho humoristique à la description d'un ancien système d'exploitation - comme un moyen rapide et sale (quick and dirty) d'introduire la philosophie de l'information (PI), et de l'imposer comme le développement le plus nécessaire de la philosophie, eu égard à son histoire. Pour évacuer cette critique, permettez-moi d'esquisser maintenant l'histoire longue qui nous lie à Turing.

Certes, ce serait une trop grande boutade que d'attribuer à Turing la fondation ou même le début d'une nouvelle philosophie de l'information. Après tout, il ne s'est jamais concentré sur le concept d'information lui-même, ni sur des problèmes de la communication, conçue comme flux d'informations ou transmission depuis Shannon, bien que chacun connaisse le travail de l'autre. Ainsi, l'index de Turing, The Essential Turing (Turing 2004), ne contient même pas d'entrée pour « information » et un livre comme Information Science (Luenberger 2006) mentionne Turing une seule fois, moins que le Parc de Bletchley. Et pourtant, je soutiendrais que sans Turing, sans son travail révolutionnaire sur le traitement de l'information, sans ses conséquences scientifiques et technologiques et sans les trois leçons décrites ci-dessus, l'intérêt contemporain pour la philosophie de l'information serait très difficile à expliquer. Turing partage avec Shannon et Wiener le mérite d'avoir attiré notre attention philosophique sur le monde de l'information et sur sa dynamique. Sans ces trois leçons, il n'y aurait aucune philosophie de l'information. Le fait que, de nos jours, nous allons plus probablement traiter les ordinateurs comme des machines de communication, plutôt que des calculatrices puissantes, et les téléphones portables comme des mini ordinateurs, donne une idée de l'influence du travail de Turing sur notre monde.

\section{Conclusion}

Le développement de nouvelles idées philosophiques semble être apparenté à l'innovation économique. Par exemple, lorsque Schumpeter a développé l'idée de « destruction créative » pour interpréter l'innovation économique, il aurait aussi pu parler du développement intellectuel. La philosophie fleurit en se reconstruisant constamment. De nos jours, ses capacités d'innovation 
et de création sont représentées et alimentées par le monde de l'information, du calcul et par les phénomènes de communication, les sciences et technologies correspondantes et les nouveaux environnements associés : la vie sociale, aussi bien que les questions existentielles, culturelles, économiques et éducatives qu'ils induisent. C'est un nouveau scénario, qui doit beaucoup au travail de Turing et à son héritage intellectuel. Dans les pages précédentes, j'ai esquissé trois leçons philosophiques que nous devrions apprendre de Turing. J'ai suggéré que la philosophie de l'information, dans la mesure où elle mène à la réalisation de l'héritage de Turing, puisse se présenter comme un paradigme novateur qui ouvre une zone très riche, utile et opportune en matière d'enquêtes conceptuelles. La PI cherche à dépasser la frontière de notre compréhension philosophique, en fournissant des méthodologies novatrices pour aborder nos problèmes les plus importants dans une perspective authentiquement contemporaine. Elle n'oublie pas l'intuition de Turing, qui insistait sur l'importance cruciale de l'abstraction pour s'assurer que les problèmes soient abordés de la meilleure façon.

La première révolution scientifique a déplacé les centres d'intérêt des philosophes du XVII ${ }^{\mathrm{e}}$ siècle de la nature de l'objet connaissable à la relation épistémique entre cet objet et le sujet du savoir, et par conséquent de la métaphysique à l'épistémologie. La croissance ultérieure de la société de l'information et l'apparition de l'infosphère, en tant qu' environnement dans lequel des millions de personnes passent de nos jours leurs vies, a poussé la philosophie contemporaine à privilégier la réflexion critique d'abord dans le domaine représenté par la mémoire et les langages de la connaissance organisée, les instruments par lesquels l'infosphère est gérée - se déplaçant ainsi de l'épistémologie à la philosophie du langage et à la logique - et ensuite à la nature de sa vraie « essence » : l'information elle-même et sa dynamique, incluant la communication, ses flux et son traitement. En conséquence, l'information a surgi comme un concept aussi fondamental et important que l'Être, la connaissance, la vie, l'intelligence, le sens ou le bien et le mal - autant de concepts centraux, dont elle dépend et qu'elle renouvelle. C'est aussi un concept qui semble plus pauvre, dans la mesure où il semble plus difficilement définissable en rapport avec les précédents. Mais c'est là que résident sa fécondité et son efficacité actuelle. C'est pourquoi la philosophie de l'information peut expliquer et guider la construction de notre environnement intellectuel et proposer un traitement systématique et efficace des fondements conceptuels de notre société contemporaine.

L'avenir de la PI dépend en grande partie de notre capacité à tirer parti de l'héritage intellectuel de Turing, en articulant les «problèmes les plus importants » de notre temps à ceux des problématiques philosophiques classiques. Étant d'un naturel optimiste, je voudrais aussi remercier 
Turing d'avoir donné une suite concrète, calculable et réalisable au projet baconien-galiléen de prise et de manipulation de l'alphabet de l'univers : c'est grâce à Turing qu'il a commencé à trouver son accomplissement dans la révolution informatique et informationnelle qui affecte désormais profondément notre connaissance de la réalité et notre manière de la conceptualiser - et par conséquent de nous conceptualiser dans ce nouveau contexte. Les récits informationnels possèdent un pouvoir ontique, non pas comme des conversations magiques, des expressions d'un logos théologique ou des formules mystiques, mais de façon immanente : à la façon des outils que nous construisons et qui finissent par décrire, modifier et mettre en œuvre notre environnement et notre propre existence, sinon sa conscience. De ce point de vue, la philosophie de l'information peut être présentée comme l'étude des activités informationnelles qui rendent possible la construction, la conceptualisation, la sémantisation (le « donner la signification à ») et finalement l'administration morale de la réalité, tant naturelle qu'artificielle, tant physique qu'anthropologique. La philosophie de l'information permet à l'humanité de saisir la signification du monde et de le construire avec sérieux. Elle promet d'être l'un des domaines de recherche philosophique les plus passionnants et les plus féconds de notre époque. Son développement sera une façon appropriée de continuer le travail de Turing et honorera son héritage dans la philosophie $^{5}$.

Traduit de l'anglais par Paolo Quintili avec la collaboration d'Éric Guichard

\section{BIBLIOGRAPHIE}

FLORIDI, L., (2008). « The Method of Levels of Abstraction », Minds and Machines 18(3): 303329.

FLORIDI, L., (2010). Information - aVery Short Introduction. Oxford, Oxford University Press. FLORIDI, L. (2011). The Philosophy of Information. Oxford, Oxford University Press. FLORIDI, L. (forthcoming). Turing Test and the Method of Levels of Abstraction. Alan Turing - HisWork and Impact. Cooper, S. B. and Leeuwen, J. V., Elsevier. FLORIDI, L., TADDEO, M., et al. (2009). «Turing's Imitation Game : Still a Challenge for Any 
Machine and Some Judges. » in Minds and Machines 19(1): 145-150.

FREUD, S. (1917). « A Difficulty in the Path of Psycho-Analysis. » in The Standard Edition of the Complete PsychologicalWorks of Sigmund Freud XVII(1917-1919) : 135-144.

LUENBERGER, D. G. (2006). Information Science. Princeton; Princeton University Press.

MOOR J. (2003). The Turing Test :The Elusive Standard of Artificial Intelligence. Dordrecht ; Kluwer Academic Publishers.

TURING, A. M. (1950). « Computing Machinery and Intelligence. » in Mind 59(236) : 433-460.

TURING, A. M. (2004). The Essential Turing : SeminalWritings in Computing, Logic, Philosophy, Artificial Intelligence and Artificial Life, Plus the Secrets of Enigma. Oxford, Clarendon Press.

WEINERT, F. (2009). Copernicus, Darwin, and Freud: Revolutions in the History and Philosophy of Science. Oxford, Blackwell.

\section{NOTES}

* Research Chair in Philosophy of Information and GPI, University of Hertfordshire ; Faculty of Philosophy and IEG, University of Oxford; UNESCO Chair in Information and Computer Ethics. Address for correspondence: Department of Philosophy, University of Hertfordshire, de Havilland Campus, Hatfield, Hertfordshire AL10 9AB, UK; 1.f1oridi@herts.ac.uk.

1. «Mars Climate Orbiter Mishap Investigation Board Phase I Report 》 (Press release). NASA: ftp://ftp.hq.nasa.gov/pub/pao/reports/1999/MC0_report.pdf.

2. Pour une introduction, voir Bibliographie: (Floridi 2010); pour une analyse plus approfondie, voir Bibliographie : (Floridi 2011).

3. Sur l'utilisation de la méthode des niveaux d'abstraction en philosophie, voir Bibliographie: (Floridi 2008) et (Floridi 2011). Sur le rôle crucial de Turing dans 1e développement de la méthode, voir Bibliographie: (Floridi forthcoming).

4. Et par conséquent la façon dont les objets philosophiques ont été infléchis une fois posées les fondations de la machine de Turing [n.d.t.].

5. Cet article est une traduction adaptée de 1'essai: Turing's Three Phi7osophical Lessons and the Philosophy of Informations, dans Royal Society's Philosophical Transactions A, Londres, 2012, 370, 3536-3542. 